\title{
Sensorless Planning for Medical Needle Insertion Procedures
}

\author{
Ron Alterovitz and Ken Goldberg \\ IEOR \& EECS Departments \\ University of California, Berkeley \\ Berkeley, CA 94720-1777, USA
}

\author{
Jean Pouliot, Richard Taschereau, and I-Chow Hsu \\ Department of Radiation Oncology \\ University of California, San Francisco \\ San Francisco, CA 94143-1708, USA
}

\begin{abstract}
Medical procedures such as seed implantation, biopsies, and treatment injections require inserting a needle tip to a specific target location inside the human body. This is difficult because (1) needle insertion causes soft tissues to displace and deform, and (2) it is often difficult or impossible to obtain precise imaging data during insertion. We are developing a sensorless planning system for needle insertion that incorporates numerical optimization with a soft tissue simulation based on a dynamic FEM formulation that models the effects of needle tip and frictional forces using a 2D mesh. In this paper we describe a sensorless planning algorithm for radioactive seed implantation that computes needle insertion offsets that compensate for tissue deformations. We apply the method to seed implantation during permanent seed prostate brachytherapy to minimize seed placement error in simulation without relying on real-time imaging.
\end{abstract}

\section{INTRODUCTION}

Medical procedures such as brachytherapy, biopsies, and treatment injections require inserting a needle to a specific target location inside the body to implant a radioactive seed, extract a tissue sample, or inject a drug. In all cases, the needle tip should be as close as possible to an internal target when the procedure is performed. Unfortunately, inserting the needle causes the surrounding soft tissues to displace and deform. Real-time imaging is often not available during insertion or is of poor quality. As illustrated using simulation in Fig. 1 left column, lack of planning can result in substantial placement error.

We are developing a sensorless planning system for needle insertion to reduce placement error. Given the target location, our system computes a needle offset that compensates for tissue deformations. We use a 2D FEM model of the soft tissues surrounding the target implant location and then use dynamic simulation of needle insertion to compute tissue deformations. The planner iteratively tests different insertion locations and depths to compute the optimal needle offset: a sensorless motion plan as illustrated in Fig. 1 right column greatly reduced placement error.

In this paper we demonstrate the system in the context of permanent seed prostate brachytherapy, a minimally invasive medical procedure that is widely used for treating prostate cancer due to the excellent long-term outcomes. Before the implant procedure, a dosimetric plan specifying desired seed locations is prepared based on static ultrasound imaging of

IEEE/RSJ International Conference on Intelligent Robots and Systems, Las Vegas, NV, October 2003. For more information, please contact ron@ieor.berkeley.edu, pouliot@radonc17.ucsf.edu, taschere@radonc17.ucsf.edu, hsu@radonc17.ucsf.edu, or goldberg@ieor.berkeley.edu, or visit http://alpha.ieor.berkeley.edu/ron/research.

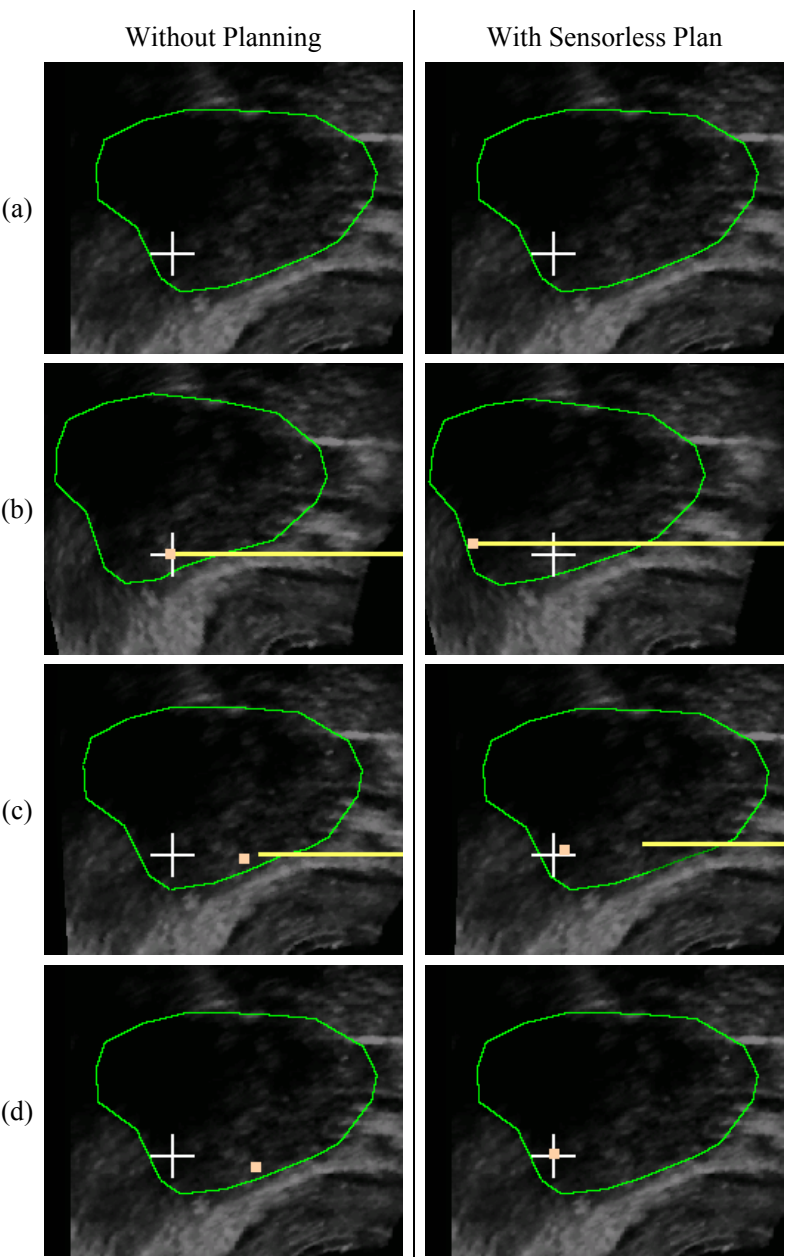

Fig. 1: Four vertical frames illustrating needle insertion based on deforming ultrasound images of the human prostate using simulation. The left column shows results without planning, producing substantial seed placement error The right column shows results with the sensorless plan, with minimal placement error. The target implant location is indicated in all frames with a white cross fixed in the external world frame. Frame (a) outlines the prostate In Frame (b), the needle is inserted and the radioactive seed (small square) is released at the needle tip. In Frame (c), the needle is retracted. Frame (d) indicates the resulting placement error, the distance between the target and resulting actual seed location. Without planning, placement error is substantial: $26 \%$ of the prostate diameter, resulting in damage to healthy tissue and failure to kill cancerous cells. With sensorless planning, shown in the right image of Frame (d), placement error is negligible.

the prostate and medical considerations to provide a high radioactive dose to cancerous cells and a low dose to healthy tissues. Numerical methods for computing optimal seed locations have been developed [23,30]. Multiple seeds and biodegradable spacers, each smaller than a grain of rice, are loaded into needles that the physician inserts transperineally 
into the patient who is lying on his back. Seeds and spacers are injected at the depth specified by the dosimetric plan. The permanently implanted radioactive seeds irradiate the surrounding tissue over several months before becoming inert.

The success of this procedure depends on the accurate placement of seeds within the prostate gland so that the desired radioactive dose distribution is achieved [12, 22]. Improper seed placement will result in an unsatisfactory dose distribution that irradiates healthy tissues and fails to kill cancerous cells. Seed placement error is the Euclidean distance between the desired location specified by the dosimetric plan (the target) and the actual implanted seed location.

Tissue deformations during needle insertion and retraction contribute to seed placement error $[22,26]$, as illustrated in Fig. 1. In [26], an experienced physician implanting seeds (without stabilizing needles) in 20 patients achieved average displacement errors of $0.47 \mathrm{~cm}$ in depth and $0.22 \mathrm{~cm}$ in height for an average placement error of $0.63 \mathrm{~cm}$, a substantial error of $21 \%$ of average prostate diameter $(3 \mathrm{~cm})$. Real-time ultrasound imaging is used during the procedure to help guide each needle along a straight path and to verify the depth of the needle tip in the world frame. However, the imaging cannot effectively be used to compensate for deformations because it does not include crisp markers with known positions inside the tissues. Below we describe a sensorless planning approach using simulation that can reduce seed placement error without relying on real-time imaging.

\section{RELATED WORK}

In robotics, sensorless planning algorithms, pioneered by Mason and Erdmann in the 1980s [14], have been developed to position and orient mechanical parts using parallel jaws [9, 16], vibrating surfaces [8], single joint robots over conveyor belts [1], and squeeze and roll primitives for micro-scale parts [20]. Sensorless planning has also been applied to manipulating objects by stable pushing [19] and insertion of parts inside fixtures [6]. For seed placement planning using needles, our goal is to model and compensate for mechanical response before actions are performed.

To apply sensorless planning to needle insertion, we require a fast and accurate simulation. DiMaio and Salcudean performed pioneering work in simulating the deformations that occur during needle insertion [13]. Their simulation, based on a quasi-static finite element method, achieves extremely fast update rates $(500 \mathrm{~Hz})$ and high accuracy (node displacement error of $1.4 \mathrm{~mm}$ for needle penetration of $70 \mathrm{~mm}$ ). High accuracy requires a calibration phase where the force distribution along the needle shaft is estimated based on observed tissue deformations. This force distribution, which is modeled with a parameterized surface in Figure 11 of [13], may be difficult to measure in vivo. Our simulation uses an alternative model based on a reduced set of scalar parameters such as needle friction, sharpness, and velocity [3]. The sensitivity of seed placement error to these parameters was analyzed in [3]. In this paper, we focus on sensorless planning: computing offsets for insertion height and depth to minimize placement error.

Needle insertion simulation requires computing deformations of soft tissue when forces are applied. The history of offline animation and real-time simulation of deformable objects is summarized in [15]. Unlike heuristic methods like the mass-spring model, the finite element method (FEM) is based on the equations of continuum mechanics. The feasibility and potential of this approach for animation was demonstrated by Terzopolous et al. [28]. Realtime visual performance for surgery simulation of the human liver using FEM was achieved by Stéphane Cotin et al., although the required preprocessing step took 8 hours on a standard PC [11]. They modeled tissue as a linearly elastic material and allowed only small quasi-static deformations.

Our simulator relaxes the quasi-static assumption and simulates dynamic deformations, as formulated by Zhuang [31] and Picinbono et al. [21]. These dynamic simulations rely on mass lumping to achieve interactive performance, but the loss of realism for soft tissues resulting from this approximation is relatively low as shown experimentally in [4]. Both Zhuang and Picinbono et al. use quadratic strain to accurately model large deformations, and $\mathrm{Wu}$ et al. extended this work to include nonlinear material elasticity [29].

Setting accurate parameters for tissue properties is important for realistic simulation. We use results from Krouskop et al., who estimated the elastic modulus for prostate and breast tissue using ultrasonic elastography [18]. Recent work in nonlinear parameter estimation includes [9] and [17]. Kataoka et al. separately measured tip and frictional forces during needle insertion into a canine prostate [25].

When real-time sensor data such as MR imaging is available during needle insertion procedures, robotic control algorithms can be used to steer the needle to the desired target [24]. However, sensorless planning based on preoperatively predicting the effects of tissue deformations must be used when real-time sensor data is not available or unreliable. In [5], a piece-wise nonlinear FEM model was used to track the position of a tumor during breast compression before a breast cancer biopsy. However, we are not aware of past work explicitly simulating needle insertion and the resulting tissue deformations to plan needle procedures without real-time sensor input.

\section{Problem Definition}

In this paper we consider the problem in the 2D plane; we are currently working on extending our approach to $3 \mathrm{D}$. As illustrated in Fig. 2, the slice of the tissue is in the $y z$ plane and the needle moves parallel to the $z$-axis at some "insertion height" $y_{r}$ to an "insertion depth" $z_{r}$. Let $\mathbf{p}_{\mathbf{t}}=\left(y_{t}, z_{t}\right)$ be a specific target point in the undeformed prostate. The point $\mathbf{p}_{\mathbf{r}}=\left(y_{r}, z_{r}\right)$ denotes the release point at the tip of the needle after insertion. (The release point would be the sample 
collection point in the case of a biopsy.) After needle retraction, the actual final location of the seed is $\mathbf{p}_{\mathbf{a}}=\left(y_{a}, z_{a}\right)$, where $\mathbf{p}_{\mathbf{a}} \neq \mathbf{p}_{\mathbf{r}}$ due to tissue deformations during needle insertion and retraction. Seed placement error is the Euclidean distance between $\mathbf{p}_{\mathbf{a}}$ and $\mathbf{p}_{\mathbf{t}}$ :

$$
\varepsilon=\left\|\mathbf{p}_{\mathbf{a}}-\mathbf{p}_{\mathbf{t}}\right\|
$$

For a given target point inside soft tissue, the problem is to compute a release point $\mathbf{p}_{\mathbf{r}}$ that minimizes placement error.

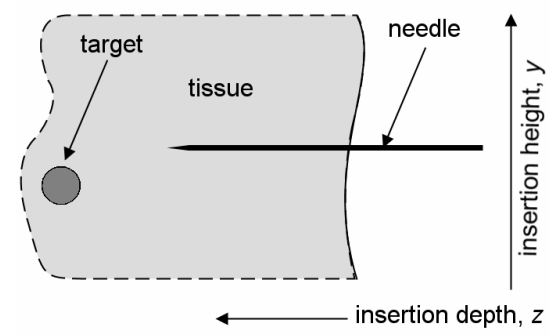

Fig. 2: Slice of deformable tissue in the $y z$ plane. The needle is inserted from right to left parallel to the $z$-axis, causing the tissue to deform.

During permanent seed prostate brachytherapy, roughly 20 bevel tip needles are each loaded with multiple seeds separated by spacers. During needle retraction, the "train" of seeds and spacers are released in the prostate. In this paper, we only address placement of the first seed in the train and ignore the remaining seeds in each needle; we plan to address multiple seeds per needle in the future. Each needle is fully retracted before the next is inserted. Hence, we assume each needle insertion and seed implantation procedure is independent. Unlike needles, we assume seeds do not cut tissue. Hence, a seed will move only when the surrounding tissue deforms. We also approximate the needle as thin and rigid with a symmetric pointed tip. Once the needle is in contact with tissue, its $y$-coordinate is fixed and it only moves parallel to the horizontal $z$-axis. We assume the needle is inserted at a constant velocity of $0.5 \mathrm{~cm} / \mathrm{sec}$.

A metal block containing approximately 50 holes at fixed coordinates is used by the physician to guide each needle during insertion. We relax the discrete insertion coordinate restriction and allow the insertion height $y_{i}$ to vary continuously, which allows for better minimization of seed placement error but will require new hardware in medical practice. The region of skin where the needle can be feasibly and safely inserted into the patient is limited, so we restrict the range of $y_{r}$ to $y_{r} \in\left(y_{\min }, y_{\max }\right)$. The maximum medically feasible insertion depth is given by $z_{\max }$.

Computing the seed placement error $\varepsilon=\left\|\mathbf{p}_{\mathbf{a}}-\mathbf{p}_{\mathbf{t}}\right\|$ requires a relation between the actual seed implant location $\mathbf{p}_{\mathbf{a}}$ and the release point $\mathbf{p}_{\mathbf{r}}$. We compute $\mathbf{p}_{\mathbf{a}}$ as a function of $\mathbf{p}_{\mathbf{r}}$ numerically using simulation. (We are not aware of a closedform equation that computes deformations in inhomogeneous tissues caused by needle insertion.)

The required simulator must estimate soft tissue deformations caused by needle insertion in $2 \mathrm{D}$ over time steps of duration $h$. We assume all soft tissues are elastic, but other properties may vary depending on tissue type and limitations imposed by the simulator used. The numerical experiments discussed in this paper use the FEM simulator described in section IV, which is an extension of past work [3], using a time step $h=1 / 30$ seconds.

The planning algorithm's inputs and outputs for each needle and target seed placement are defined by:

Input:

Needle insertion simulator with required parameters (as defined in section IV)

$\mathbf{p}_{\mathbf{t}}$ : Target coordinate in the tissue

$\left(y_{\min }, y_{\max }\right)$ : Range of feasible insertion heights

$z_{\text {max }}$ : Maximum feasible insertion depth

$v$ : Needle velocity during insertion and retraction

$h$ : Simulation time step

Output:

$\mathbf{p}_{\mathbf{r}}{ }^{*}$ : Release point that minimizes seed placement error

A naïve planner that ignores tissue deformations would set $\mathbf{p}_{\mathbf{r}}=\mathbf{p}_{\mathbf{t}}$. To estimate an optimal release point $\mathbf{p}_{\mathbf{r}}{ }^{*}$, the planner computes an offset from $\mathbf{p}_{\mathbf{t}}$ for both the insertion depth and height. The offset for needle insertion depth is necessary because tissue in front of the needle tip is compressed during insertion; the needle must be inserted deeper than $z_{t}$ to compensate for this compression. The offset for insertion height is necessary since organs or glands (such as the prostate) may rotate during needle insertion. For example, if the target is located near the bottom of the prostate, inserting the needle near the target height causes the prostate to rotate slightly clockwise, as shown in Fig. 3. The needle must be inserted higher to compensate for its deflected path through the prostate. This occurs because the prostate is composed of a stiffer material than the surrounding soft tissue. Hence, both needle insertion depth and height must be planned to minimize placement error.

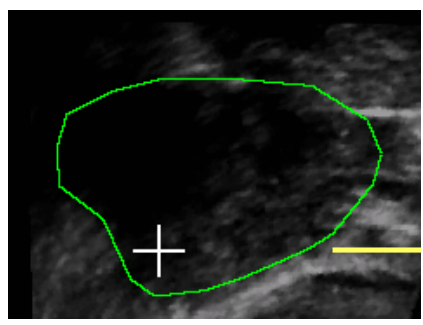

(a) Needle approaches prostate

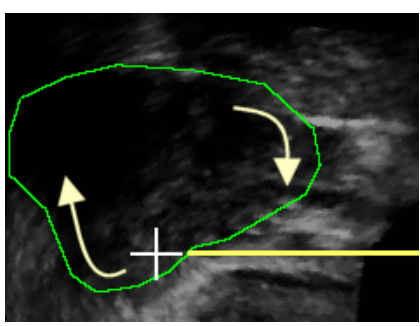

(b) Prostate rotated by needle
Fig. 3: When the needle pushes against the lower half of the prostate from the right, the prostate rotates clockwise slightly because it is stiffer than the surrounding tissue. This slight rotation can lead to significant changes in the optimal needle insertion height.

\section{NEEDLE INSERTION SIMULATION}

Our simulator of $2 \mathrm{D}$ elastic soft tissue deformations is based on [3]. We approximate soft tissues as linearly elastic materials. Tissue may be inhomogeneous but must be fully connected with no gaps between different tissue types. 


\section{A. Simulation Input}

The geometry of the soft tissue surrounding the target is defined using a reference mesh composed of $m$ discrete 3node triangular elements created using $n$ total nodes, each with 2 degrees of freedom. Each element in the mesh may be assigned unique material properties, which allows for the simulation of inhomogeneous tissue. As specified in [3], the simulator uses biomechanical values for tissue stiffness (Young's modulus) and compressibility (Poisson ratio) and for needle properties specified by the coefficients of static and kinetic friction and the force required to cut a unit length of soft tissue.

\section{B. Simulation Output}

The simulation computes mesh deformations that simulate the tissue's response to the needle over time. The reference mesh defines the geometry of the tissue, with each node's coordinate stored in the position vector $\mathbf{x}$. The deformed mesh is constructed using the node coordinates $\mathbf{x}+\mathbf{u}_{\mathbf{i}}$ in the world frame, where $\mathbf{u}_{\mathbf{i}}$ is the nodal displacement vector computed for each time step $i$. Using a fixed time step duration $h$, we obtain simulated deformations for times $t=h i$, $i \geq 0$.

The coordinate of a node $k$ in the world frame at time step $i$ is given by $\mathbf{x}_{\mathbf{k}}+\mathbf{u}_{\mathbf{i}, \mathbf{k}}$ where $\mathbf{x}_{\mathbf{k}}$ is the reference mesh coordinate and $\mathbf{u}_{\mathbf{i}, \mathbf{k}}$ is the displacement of node $k$ at time step $i$. As shown in Fig. 4 , the coordinate of a target point $\mathbf{p}_{\mathbf{t}}$ located at node $k$ in the reference mesh is displaced by $\mathbf{u}_{\mathbf{i}, \mathbf{k}}$ when the mesh is deformed at time step $i$. (The displacement of target points not located at nodes can be computed in $O(1)$ time using interpolation of the displacements of the nodes of the enclosing element.)

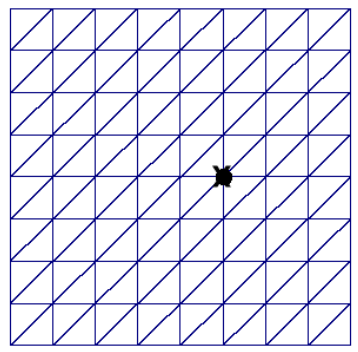

(a) Initial mesh

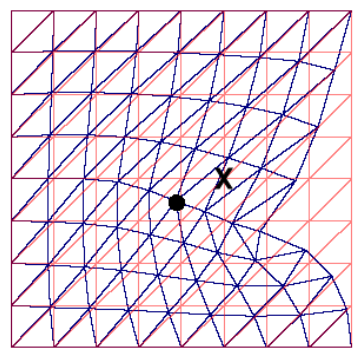

(b) Deformed mesh
Fig. 4: At time step $i=0, \mathbf{u}_{0}=0$ and the coordinate of the target in the reference mesh $\mathbf{x}_{\mathbf{k}}$, denoted by $\times$, coincides with the target in the world frame, denoted by $\cdot$ (a). Both the $\times$ and $\cdot$ represent the same location within the tissue, but their world frame coordinates differ by $\mathbf{u}_{\mathbf{i}, \mathbf{k}}$ when the tissue is deformed at time step $i>0$ (b).

\section{Computing Soft Tissue Deformations}

We use a finite element method (FEM) to compute the deformations of soft tissue when forces are applied by the needle. For realistic results, we simulate the dynamic behavior of the tissue by solving for the acceleration, velocity, and displacement of each node at every time step.

Using the standard notation in [32], the FEM problem for mesh the given reference mesh is defined by a system of up to $d=2 n$ linear differential equations:

$$
\mathbf{M} \mathbf{a}_{\mathrm{i}}+\mathbf{C} \mathbf{v}_{\mathrm{i}}+\mathbf{K} \mathbf{u}_{\mathrm{i}}=\mathbf{f}_{\mathrm{i}}
$$

where $\mathbf{M}$ is the mass matrix, $\mathbf{C}$ is the damping matrix, $\mathbf{K}$ is the stiffness matrix, and at time step $i, \mathbf{f}_{\mathrm{i}}$ is the external force vector, $\mathbf{a}_{\mathrm{i}}$ is the nodal acceleration vector, $\mathbf{v}_{\mathrm{i}}$ is the nodal velocity vector, and $\mathbf{u}_{\mathrm{i}}$ is the nodal displacement vector.

To integrate the differential system (1) over time, we use the Newmark method, which translates the differential system into a linear system of equations which we solve using the methods in [3] for interactive performance.

\section{Simulating Needle Insertion}

We simulate the force the needle exerts on the tissue at its tip and frictional forces along the needle shaft. These forces applied by the needle are computed and the FEM force vector $\mathbf{f}_{\mathrm{i}}$ is updated at every time step.

Using FEM, forces are applied as boundary conditions on elements in the reference mesh. Since the needle may be inserted at any location, it is usually necessary to modify the reference mesh in real-time to ensure that element boundaries are present where the tip and friction forces must be applied. To apply the tip force, a node is maintained at the needle tip location during insertion. To apply the friction forces, a list of nodes along the needle shaft is maintained and these nodes are constrained to only move horizontally along the needle shaft. These mesh modifications, which modify the reference mesh coordinate vector $\mathbf{x}$, are fully described in [3].

Nodes along the needle shaft also carry state information; they are either attached to the needle (in static friction state) or allowed to slide along the needle shaft (in dynamic friction state). State transitions are determined by the friction coefficients. The node state information and displacement computation produce a history dependant simulation where the output of time step $i$ depends on the output of time step $i-1$.

A seed can be implanted at the location of the needle tip in the reference mesh at any insertion depth. After implantation, we assume the seed's reference mesh coordinate is fixed and it moves in the world frame only as a result of surrounding tissue deformations. The location of the seed in the world frame is tracked efficiently as described in [3].

The computation time required for each simulation time step depends on the numerical method used to solve equation (1). For fastest performance, we use an $O(n)$ method as described in [3].

\section{NeEdle Insertion Planning}

Given a target point $\mathbf{p}_{\mathbf{t}}$, the goal of needle insertion planning is to find an optimal release point $\mathbf{p}_{\mathbf{r}}{ }^{*}$ that minimizes seed placement error $\varepsilon=\left\|\mathbf{p}_{\mathbf{a}}-\mathbf{p}_{\mathbf{t}}\right\|$, where the actual seed implant location $\mathbf{p}_{\mathbf{a}}$ is a function of the release point $\mathbf{p}_{\mathbf{r}}$. The optimal $\mathbf{p}_{\mathbf{r}}{ }^{*}$ cannot be computed analytically by differentiating the error function $\varepsilon$ because the relationship between $\mathbf{p}_{\mathbf{r}}$ and $\mathbf{p}_{\mathbf{a}}$ can only be computed numerically by simulation. Instead, our algorithm efficiently uses simulation 
to estimate the optimal $\mathbf{p}_{\mathbf{r}}{ }^{*}$.

We first describe how to efficiently compute $\mathbf{p}_{\mathbf{a}}$ and $\varepsilon$ using simulation when given the release point $\mathbf{p}_{\mathbf{r}}=\left(y_{r}, z_{r}\right)$. Then, we propose a method to estimate the optimal depth $z_{r}{ }^{*}$ that minimizes the error $\varepsilon$ when a candidate insertion height $y_{r}$ is given. Finally, we describe how to select candidate insertion heights to estimate the optimal $y_{r}{ }^{*}$ that minimizes $\varepsilon$.

\section{A. Computing Placement Error Given the Release Point}

Given the target $\mathbf{p}_{\mathbf{t}}$ and the release point $\mathbf{p}_{\mathbf{r}}=\left(y_{r}, z_{r}\right)$, the placement error $\varepsilon=\left\|\mathbf{p}_{\mathbf{a}}-\mathbf{p}_{\mathbf{t}}\right\|$ requires an estimate of $\mathbf{p}_{\mathbf{a}}$, the actual seed location after needle insertion and retraction using a release point of $\mathbf{p}_{\mathbf{r}}$. We compute this estimate using simulation. Initially, the needle tip is outside the body, which corresponds to $z<0$. We set the insertion height $y_{r}$ and simulate needle insertion to a depth of $z_{r}$ and implant a seed in the mesh. We then simulate needle retraction until $z_{r}<0$, wait for steady state, and then use the final seed location as an estimate for $\mathbf{p}_{\mathbf{a}}$.

The time required to estimate $\mathbf{p}_{\mathbf{a}}$ can be reduced significantly by replacing the simulation of needle retraction with an $O(1)$ time computation. The simulation algorithm described in section IV.D always maintains a node at the location the needle tip during insertion. Assume the needle tip, located at node $j$, has reached the release point $\mathbf{p}_{\mathbf{r}}$ at time step $i$ in the FEM simulation. As described in section IV, both the reference mesh coordinate $\mathbf{x}_{\mathbf{k}}$ and displacement $\mathbf{u}_{\mathbf{i}, \mathbf{k}}$ for node $k$ at time step $i$ are known after the FEM system of equations has been solved. By the elasticity assumption, the displacement vector $\mathbf{u}_{\mathbf{j}}$ will be $\mathbf{0}$ for all iterations $j$ at steady state after needle retraction because no external forces are being applied to the soft tissue. Hence, the location in the world frame of the release point $\mathbf{p}_{\mathbf{r}}$ after needle retraction will be $\mathbf{x}_{\mathbf{k}}+\mathbf{u}_{\mathbf{j}}=\mathbf{x}_{\mathbf{k}}$. Since we assumed that seeds do not cut tissue, the final seed location is $\mathbf{p}_{\mathbf{a}}=\mathbf{x}_{\mathbf{k}}$ and the error is $\varepsilon=\left\|\mathbf{x}_{\mathbf{k}}-\mathbf{p}_{\mathbf{t}}\right\|$, where $\mathbf{x}_{\mathbf{k}}$ is the reference mesh coordinate of the node $k$ at the needle tip when it reaches the release point $\mathbf{p}_{\mathbf{r}}$ in simulation.

\section{B. Minimizing Placement Error Given Insertion Height}

When given a candidate needle insertion height $y_{r}$, we simulate needle insertion at height $y_{r}$ to find the insertion depth $z_{r}^{*}$ that minimizes placement error $\varepsilon$.

$$
z_{r}^{*}=\arg \min \varepsilon\left(z_{r} \mid y_{r}\right) \text {. }
$$

Because the simulation of needle insertion is history dependent, we must compute $\varepsilon\left(z \mid y_{r}\right)$ in order to compute $\varepsilon\left(z+d z \mid y_{r}\right)$ for $d z, z>0$. We begin with the needle located outside the body $(z=0)$ and simulate needle insertion at height $y_{r}$ until $z=z_{\text {max }}$. Using the algorithm described in section IV, $z$ is increased by $d z=v h$ every time step, where $h$ is the time step duration and $v$ is the needle velocity. The insertion depth $z$ at each time step serves as a candidate for the optimal release point $z_{r}{ }^{*}$. We compute $\varepsilon\left(z \mid y_{r}\right)$ in $O(1)$ time as described in section V.A for every time step and save the $z_{r}{ }^{*}$ for which $\varepsilon$ is smallest. Because $\varepsilon$ is computed for every feasible insertion depth, the optimal $z_{r}^{*}$ for the given $y_{r}$ is guaranteed to be found (within a resolution of $d z$ ) regardless of the convexity properties of the function $\varepsilon\left(z_{r} \mid y_{r}\right)$.

This optimization computes $z_{\max } /(v h)$ simulation time steps, each requiring $O(n)$ time as described in section IV.D. Since the needle tip will move a distance $v h$ each time step, the resolution of $z^{*}$ is $v h$. A small time step $h$ is desirable to improve the resolution of $z^{*}$, but the number of time steps required to compute the optimal insertion depth $z^{*}$ grows as $h$ decreases.

\section{Estimating Insertion Height to Minimize Placement Error}

For any candidate insertion height $y_{r}$, we compute the optimal insertion depth $z_{r}{ }^{*}$ and the resulting placement error:

$$
\varepsilon\left(y_{r}\right)=\min \varepsilon\left(z_{r} \mid y_{r}\right)
$$

Our goal is to minimize $\varepsilon\left(y_{r}\right)$ over the given feasible range $y_{\min } \leq y_{r} \leq y_{\max }$. The value of $y_{r}{ }^{*}$

$$
y_{r}{ }^{*}=\arg \min \varepsilon\left(y_{r}\right)
$$

combined with the corresponding optimal $z_{r}{ }^{*}$

$$
z_{r}^{*}=\arg \min \varepsilon\left(z_{r} \mid y_{r}^{*}\right)
$$

specifies the optimal release point $\mathbf{p}_{\mathbf{r}}{ }^{*}=\left(y_{r}^{*}, z_{r}^{*}\right)$ that minimizes seed placement error $\varepsilon$.

Minimizing $\varepsilon\left(y_{r}\right)$ is difficult because derivative values are not available and the function is not guaranteed to be unimodal (strictly quasiconvex). In general, an approximate minimum of $\varepsilon\left(y_{r}\right)$ can be found using a grid search over $y_{r} \in\left(y_{\min }, y_{\max }\right)$.

However, the function $\varepsilon\left(y_{r}\right)$ for some simulations will be unimodal near the minimum. In particular, this property will hold when it is not possible to insert the needle at different heights and still reach the same point in the reference mesh of the tissue. Although this property is not guaranteed, it holds for most feasible targets in our simulation that are not adjacent to an inhomogeneous tissue boundary. In such cases, a line search method can be used over the feasible range to find the optimal $y_{r}{ }^{*}$. We use the golden section search method [7] because, unlike a standard binary search, it does not rely on derivative information (which is not available in the simulation). Golden section search is a variant of the Fibonacci search that requires fewer error function evaluations. Each iteration of the line search evaluates the error function $\varepsilon\left(y_{r}\right)$ for a new candidate insertion height $y_{r}$, which is computationally expensive to evaluate since it requires simulating needle insertion at height $y_{r}$ to find an optimal depth $z_{r}{ }^{*}$ and resulting error, as described in section V.B. The algorithm iterates until the optimal is found within a specified tolerance; convergence is guaranteed if the error function is unimodal.

\section{Planning for Seed Placement}

Fig. 1 provides a simulated case study showing that deformations can produce significant errors in final seed placements during prostate brachytherapy. Seed placement error should be minimized to achieve the desired radioactive dose distribution. Below we demonstrate the performance of the planner for seed placement during prostate brachytherapy. 


\section{A. Prostate Model}

Our prostate model is based on an anonymous patient who underwent brachytherapy treatment for prostate cancer at the UCSF Medical Center in June 2002. A pre-procedure ultrasound image of the prostate was superimposed as a texture map on a square planar mesh composed of 1250 triangular elements. A polygon outlining the prostate membrane was manually drawn on the texture map by a physician (coauthor I-Chow Hsu); underlying mesh elements within this polygon were assigned prostate tissue properties and the remaining elements were assigned lower stiffness fatty tissue properties, resulting in an inhomogeneous mesh. The Young's modulus and Poisson ratio were set using [18]. The remaining parameters in our simulation were set using ultrasound imaging data from a real medical procedure performed on the same patient in June 2002, as described in [1] and [3].

\section{B. Target Test Case}

We test our planning algorithm for the target $\mathbf{p}_{\mathbf{t}}=(1.50 \mathrm{~cm}$, $3.00 \mathrm{~cm}$ ) shown in Fig. 1 for a $3 \mathrm{~cm}$ diameter prostate. Without planning, deformations are ignored and the needle is inserted with zero offsets to $\mathbf{p}_{\mathbf{r}}=\mathbf{p}_{\mathbf{t}}$. Based on our simulation, this results in the seed being implanted at $\mathbf{p}_{\mathbf{a}}=(1.41 \mathrm{~cm}, 2.21 \mathrm{~cm})$, a placement error of $\varepsilon=0.79 \mathrm{~cm}$ ( $26 \%$ of the prostate diameter).

\section{Optimizing Insertion Depth}

For insertion of a needle at the target height $y_{r}=y_{t}=1.5 \mathrm{~cm}$, we plot the placement error $\varepsilon\left(z_{r} \mid y_{r}=y_{t}\right)$ in Fig. 5. The insertion depth $z_{r}=z_{t}=3.0 \mathrm{~cm}$ yields a placement error of $\varepsilon=0.79 \mathrm{~cm}, 26 \%$ of prostate diameter. The error in the depth coordinate is caused primarily because the tissue in front of the needle tip is being compressed before it is cut. Hence, the needle must be inserted deeper than the target depth to decrease the error. To minimize $\varepsilon$ for $y_{r}=y_{t}$, the needle should be inserted to a depth of $z_{r}{ }^{*}=3.84 \mathrm{~cm}$, which reduces the error by $82 \%$ to only $\varepsilon=0.14 \mathrm{~cm}, 5 \%$ of prostate diameter.

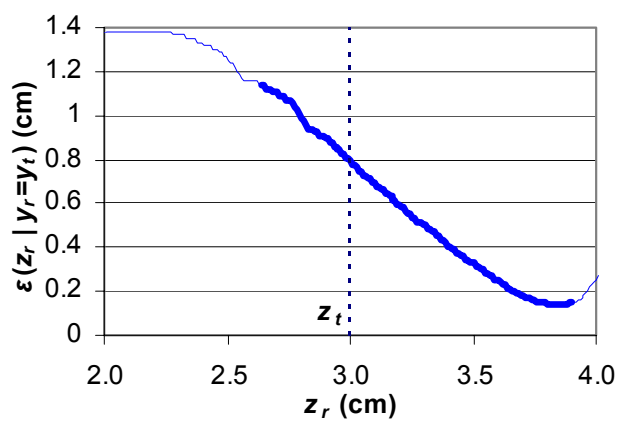

Fig. 5: Needles should generally be inserted deeper than the target depth to compensate for tissue deformations and minimize placement error. The bold portion of the line denotes feasible seed placements inside the prostate.

\section{Optimizing Insertion Height}

For a $0.6 \mathrm{~cm}$ range around the target height $y_{t}=1.5 \mathrm{~cm}$, we plot the estimated optimal insertion depth in Fig. 6(a) and the resulting error $\varepsilon$ in Fig. 6(b). The planner finds $\mathbf{p}_{\mathbf{r}}{ }^{*}$ with $\varepsilon^{*}=0.003 \mathrm{~cm}(0.1 \%$ of prostate diameter $)$ by inserting at height $y_{r}{ }^{*}=1.59 \mathrm{~cm}$ to a depth $z_{r}{ }^{*}=3.80 \mathrm{~cm}$.

To test planner performance, we selected 12 sample points inside the prostate, shown by the crosses in the Fig. 7. We apply golden section search in the range $y_{r} \in\left(y_{t}\right.$ $0.2 \mathrm{~cm}, y_{t}+0.2 \mathrm{~cm}$ ) with tolerance $0.01 \mathrm{~cm}$ for each target. Without planning, the average error was $0.59 \mathrm{~cm}(20 \%$ of prostate diameter) with a standard deviation of $0.10 \mathrm{~cm}$. Using our planner, the average error was reduced in simulation to $0.002 \mathrm{~cm}(0.07 \%$ of prostate diameter $)$ with a standard deviation of $0.004 \mathrm{~cm}$.

Our needle insertion planner and simulator were implemented using $\mathrm{C}++$ and tested on a $750 \mathrm{MHz}$ Pentium III PC with 256MB RAM. The average time to compute optimal depth given height was 8.5 seconds and computing both optimal height and depth for each target took an average of 97.7 seconds.

(a)

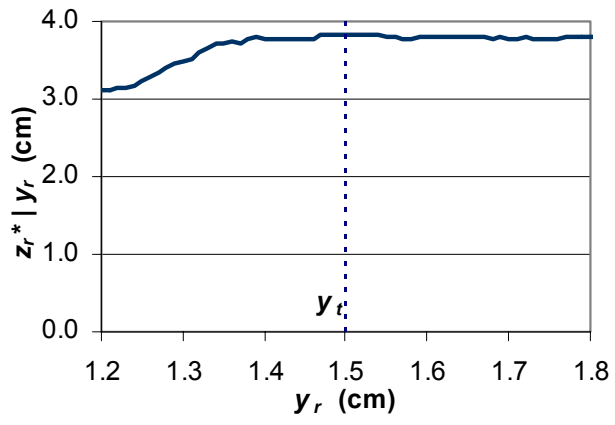

(b)

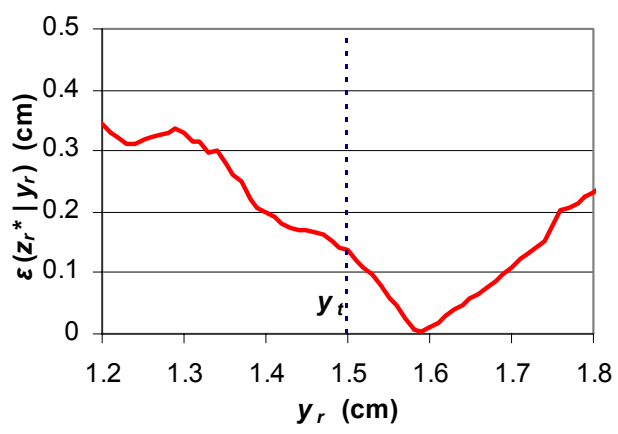

Fig. 6: For all candidate insertion heights $y_{r}$, optimal depth $z_{r}^{*}$ (a) and resulting error (b) are computed. Placement error is negligible for $\mathbf{p}_{\mathbf{r}}=(1.59 \mathrm{~cm}, 3.80 \mathrm{~cm})$.

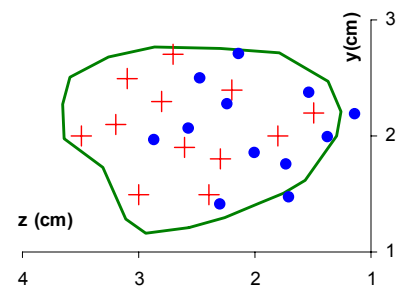

(a) Placements without planning

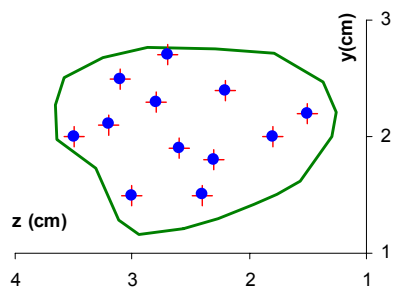

(b) Placements using planner Fig. 7: Twelve sample points were selected at random as targets maked "+" inside the prostate. Actual seed placements using simulation are marked "•". Lack of planning results in major placement errors averaging $20 \%$ of the prostate diameter (a), which will lead to a poor radioactive dose distribution. Seed placement error was neglible using the planner (b). 


\section{CONClusions}

We describe a sensorless planning method for needle insertion procedures. The method combines numerical optimization with soft tissue simulation. The simulation, based on a dynamic FEM formulation, models the effects of needle tip and frictional forces on soft tissues defined by a 2D mesh. Our sensorless planning method for radioactive seed implantation computes needle offsets to minimize seed placement error by compensating for predicted tissue deformations.

The effectiveness of the planner in vivo will be dependant on the accuracy of the simulation of needle insertion and tissue deformations for a specific patient. The sensitivity analysis in [3] suggests that 2D seed placement error in homogeneous tissues is more sensitive to physiciancontrolled parameters than to patient-specific parameters such as the Young's modulus and Poisson ratio, which is beneficial since the latter are more difficult to estimate before the procedure. We are currently investigating 3D inhomogeneous tissue models, needle bending during insertion, the interaction of multiple seeds per needle, and faster placement error minimization methods.

\section{ACKNOWLEDGEMENTS}

We thank Russ Taylor for bringing this problem to our attention and Tim Salcudean, Simon DiMaio, Allison Okamura, A. Frank van der Stappen, K. Gopalakrishnan, Dezhen Song, Han-Wen Nienhuys, and Katja Langen for their valuable feedback. We also thank the Department of Defense for their financial support through the National Defense Science and Engineering Graduate Fellowship program.

\section{REFERENCES}

[1] S. Akella, W. Huang, K. M. Lynch, and M. T. Mason, "Sensorless parts feeding with a one joint robot," In Workshop on the Algorithmic Foundations of Robotics, July 1996.

[2] R. Alterovitz, J. Pouliot, R. Taschereau, I.C. Hsu, and K. Goldberg, "Simulating needle insertion and radioactive seed implantation for prostate brachytherapy," Medicine Meets Virtual Reality 11, J.D. Westwood et al. (Eds.), IOS Press, January 2003, pp. 19-25.

[3] R. Alterovitz, J. Pouliot, R. Taschereau, I.C. Hsu, and K. Goldberg, "Needle insertion and radioactive seed implantation in human tissues: simulation and sensitivity analysis," in Proc. of the IEEE Int. Conf. on Robotics and Automation, September 2003.

[4] R. Alterovitz and K. Goldberg, "Comparing algorithms for soft tissue deformation: accuracy metrics and benchmarks," June 2002. Available: http://alpha.ieor.berkeley.edu/ron/research/

[5] F. S. Azar, D. N. Metaxas, M. D. Schnall, "A deformable finite element model of the breast for predicting mechanical deformations under external perturbations," Academic Radiology, Vol. 8, No. 10, 2001.

[6] D.J. Balkcom, E.J. Gottlieb, J. C. Trinkle, "A sensorless insertion strategy for rigid planar parts," in Proc. IEEE Int. Conf. on Robotics and Automation, May 2002, pp. 882-7.

[7] M.S. Bazaraa, H.D. Sherali, and C.M. Shetty. Nonlinear Programming. Theory and Algorithms, Second Edition. John Wiley \& Sons, Inc. 1993.

[8] K.-F. Böhringer, V. Bhatt, B. R. Donald, and K. Goldberg, "Algorithms for sensorless manipulation using a vibrating surface," Algorithmica, 2000, 26: 389-429.
[9] Brost R.C., "Automatic grasp planning in the presence of uncertainty," Int. Journal of Robotics Research, vol.7, no.1, Feb. 1988, pp.3-17.

[10] J. Brown, J. Rosen, Y. S. Kim, L. Chang, M. N. Sinanan, B. Hannaford, "In-vivo and in-situ compressive properties of porcine abdominal soft tissues," Medicine Meets Virtual Reality 11, J.D. Westwood et al. (Eds.), IOS Press, January 2003, pp. 16-32.

[11] S. Cotin, H. Delingette, and N. Ayache, "Real-time elastic deformations of soft tissues for surgery simulation," IEEE Trans. on Visualization and Computer Graphics, Vol. 5, No. 1, 1999.

[12] J. E. Dawson, T. Wu, T. Roy, J. Y. Gy, and H. Kim, "Dose effects of seeds placement deviations from pre-planned positions in ultrasound guided prostate implants," Radiol. Oncol. 32, 1994, pp. 268-270.

[13] S. P. DiMaio and S. E. Salcudean, "Needle insertion modeling and simulation," in Proc. of the IEEE Int. Conf. on Robotics and Automation, May 2002, pp. 2098-2105.

[14] M. Erdmann and M. Mason, "An exploration of sensorless manipulation," IEEE Journal of Robotics and Automation, Vol. 4, No. 4, August, 1986, pp. 369 - 379.

[15] S. F. Gibson and B. Mirtich. "A survey of deformable modeling in computer graphics," MERL, TR-97-19, 1997.

[16] K. Y. Goldberg, "Orienting polygonal parts without sensing," Algorithmica, 1993, 10: 201-255.

[17] D. Kalanovic, M. P. Ottensmeyer, J. Gross, G. Buess, S. L. Dawson, "Independent testing of soft tissue visco-elasticity using indentation and rotary shear deformations," Medicine Meets Virtual Reality 11, J.D. Westwood et al. (Eds.), IOS Press, January 2003, pp. 137-143.

[18] T. A. Krouskop, T. M. Wheeler, F. Kallel, B. S. Garria, and T. Hall, "Elastic moduli of breast and prostate tissues under compression," Ultrasonic Imaging, Vol. 20, 1998.

[19] K. M. Lynch, "Locally controllable manipulation by stable pushing," IEEE Trans. on Robotics and Automation, 15(2):318-327, April 1999.

[20] M. Moll, K. Goldberg, M. Erdmann, R. Fearing, "Orienting micro-scale parts with squeeze and roll primitives," IEEE Int. Conf. on Robotics and Automation, May 2002.

[21] G. Picinbono, H. Delingette, N. Ayache, "Nonlinear and anisotropic elastic soft tissue models for medical simulation," in Proc. of the IEEE Int. Conf. on Robotics and Automation, May 2001, pp. 1370-1375.

[22] J. Pouliot, R. Taschereau, C. Coté, J. Roy, and D. Tremblay, "Dosimetric aspects of permanents radioactive implants for the treatment of prostate cancer," Physics in Canada 55(2), 1999, pp. 61-68.

[23] J. Pouliot, D. Tremblay, J. Roy, and S. Filice, "Optimization of permanent 125I prostate implants using fast simulated annealing," Int. J. Radiat. Onco. Biol. Phys. 36(3), 1996, pp. 711-720.

[24] M. Shi, Hong L., and G. Tao, "A stereo-fluoroscopic image-guided robotic biopsy scheme," IEEE Trans. on Control Systems Technology, Vol. 10, No. 3, May 2002, pp. 309-317.

[25] H. Kataoka, T. Washio, K. Chinzei, K. Mizuhara, C. Simone, and A. M. Okamura, "Measurement of the tip and friction force acting on a needle during penetration," Medical Image Computing and Computer Assisted Intervention, Sept. 2002.

[26] R. Taschereau, J. Pouliot, J. Roy, D. Tremblay, "Seed misplacement and stabilizing needles in transperineal permanent prostate implants," Radiotherapy and Oncology 55, 2000, pp. 59-63.

[27] R. Taschereau, J. Roy, and J. Pouliot, "Monte Carlo simulations of prostate implants to improve dosimetry and compare planning methods," Med. Phys. 26 (9), Sept. 1999.

[28] D. Terzopolous, J. Platt, A. Barr, K. Fleischer, "Elastically deformable models," Computer Graphics, Vol. 21, No. 4, 1987.

[29] X. Wu, M.S. Downes, T. Goktekin, and F. Tendick, "Adaptive nonlinear finite elements for deformable body simulation using dynamic progressive meshes," Eurographics 2001, Computer Graphics Forum, Vol. 20, No. 3, Sept. 2001, pp. 349-358.

[30] M. Zaider et al., "Treatment planning for prostate implants using magnetic-resonance spectroscopy imaging," Int. J. Radiation Oncology Biol. Phys., Vol. 47, No. 4, 2000.

[31] Y. Zhuang, "Real-time simulation of physically realistic global deformations," Ph.D. thesis, UC Berkeley, 2000.

[32] O.C. Zienkiewicz and R.L. Taylor, The Finite Element Method, Fifth Edition, Butterworth-Heinemann, 2000. 